$$
\text { CONF-970586--1 }
$$

\title{
Role of Dopant Counter-Anion Functionality in Polyaniline Salts / Blends and Implications to Morphology
}

\author{
Alan R. Hopkins and Paul G. Rasmussen \\ Center for Macromolecular Science and Engineering and Department of \\ Chemistry, The University of Michigan, Ann Arbor, MI 48109-1055
}

\section{Rafil A. Basheer \\ Polymers Department, General Motors Research and Development Center, Warren, MI 48090-9055}

\author{
B. K. Annis and G. D. Wignall \\ Oak Ridge National Laboratory, \\ Oak Ridge, TN 37831 -6393
}

\section{Introduction}

Polyanilines are of particular current interest primarily due to their relative ease of synthesis, low cost and stable conductivity in air. The insulating, polyaniline emeraldine base (PANI-EB) form becomes electrically conducting by preferential protonation or 'doping' the imine nitrogen sites to yield an electrically conducting polyaniline emeraldine salt (PANI-ES). Conductivity values of polyanilines range from insulating to $300 \mathrm{~S} / \mathrm{cm}$ and are both dopant and concentration sensitive. Like most other organic conducting polymers, the PANI-ES form suffers from limited solubility in common solvents. Cao etc. al. ${ }^{1}$ recently found that this limited solubility can be overcome by functionalizing the dopant counter-anion with polar and non-polar analogs to promote solubility in common solvents. These functionalized anions act as both flexiblizers and surfactants to the stiff polyaniline backbone to result in a soluble and processable form of PANI-ES. In addition to counter-anions providing surfactant activity, the structure of the dopant counter-anion may influence the nature of how the charged polyaniline chains stack into crystal structures, which in turn, affects the bulk electrical conductivity of the PANI-ES.

Another barrier to the use of these materials is that their doped form, when cast from solution, is inherently brittle, and thus must be blended with another polymer to be useful in most industrial applications. In PANI-ES, the onset of electrical conductivity occurs at low volume fractions of the conductive component, compared to metal filled composites. ${ }^{2}$ This is primarily due to the unique morphology of the PANI-ES that is formed within the insulating host polymer. PANI-ES forms a network structure at volume fractions of $<1 \%{ }^{3}$ The mechanism behind the formation of this immiscible polymer network may be due, in part, to the polarity differences (induced by the functionality of the dopant counter-anion) in the doped polyaniline and the insulating host polymer. This phase separation of PANI-ES and an insulating matrix into a cellular-like network is central to the idea of obtaining high conductivities at low salt loading fractions.

In this paper, wide and small angle $X$-ray scattering techniques (i.e. WAXS and SAXS) and light microscopy are used to characterize the influence of the dopant's structure on the morphology of both polyaniline sait and blend. In an attempt to modify the morphology of the PANI-ES, we have evaluated a number of doping acids (i.e. methane sulfonic acid (HMSA), butane sulfonic acid (HBSA), dodecyl benzene sulfonic acid (HDBSA) and camphor sulfonic acid (HCSA)) that vary in size and polarity to better understanding the role of the acid in PANI-ES's morphology and 


\section{DISCLAIMER}

This report was prepared as an account of work sponsored by an agency of the United States Government. Neither the United States Government nor any agency thereof, nor any of their employees, make any warranty, express or implied, or assumes any legal liability or responsibility for the accuracy, completeness, or usefulness of any information, apparatus, product, or process disclosed, or represents that its use would not infringe privately owned rights. Reference herein to any specific commercial product, process, or service by trade name, trademark, manufacturer, or otherwise does not necessarily constitute or imply its endorsement, recommendation, or favoring by the United States Government or any agency thereof. The views and opinions of authors expressed herein do not necessarily state or reflect those of the United States Government or any agency thereof. 


\section{DISCLAMMER}

Portions of this document may be illegible in electronic image products. Images are produced from the best available original document. 
polycaprolactam using hexafluoro-2-propanol (HFIP) as a solvent. Our other goal was to investigate the effect of the counter-anion structure on the nature of the phase separated PANI-ES network. The shape of the PANIES network in the host polycaprolactam has important implications on the nature of conduction behavior and the final electrical conductivity of the blend.

\section{Experimental}

Synthesis: Polyaniline emeraldine base (PANI-EB) was prepared via the $\mathrm{FeCl}_{3}$ method as describedelsewhere. ${ }^{4}$

Doping: Doped polyaniline solutions were prepared in hexafluoro-2-propanol (HFIP) by a solution doping method. Solutions tumed from blue / brown color to a forest green color, characteristic of doped polyaniline. Molar doping of undoped polyaniline is calculated for polyaniline emeraldine salt from the mole ratio $y=$ (moles of dopant) / (moles of phenyl-NH), determined by elemental analysis. Optimally doped polyaniline has the value of $y=0.5$. Dopants used were: camphor sulfonic acid (HCSA), Aldrich; methane sulfonic acid (HMSA), Aldrich; butane sulfonic acid (HBSA), Eastman Kodak and dodecyl benzene sulfomic acid (HDBSA), TCI America. Polycaprolactam (Aldrich) was vacuum dried before solution blending. All PANI-ES solutions were filtered with a $0.50 \mu \mathrm{m}$ filter.

Film preparation: All filtered, doped polyaniline salt and blend solutions were solution cast onto a Teflon coated glass substrate. They were covered with a glass dish to allow for slow evaporation (over a period of 24 hours) of the solvent at room temperature. HFIP, with a boiling point of $60^{\circ} \mathrm{C}$, allows gentle stripping at room temperature. All films were peeled off the Teflon substrate and dried in a dynamic vacuum oven at $75{ }^{\circ} \mathrm{C}$ for approximately 24 hours. From fluorine elemental analysis, percent residual HFIP solvent in films was less than $0.5 \%$ (wt/wt). This casting process from HFIP procucod robust, free standing and solvent free polyaniline salt and blend films.

WAXS and SAXS data acquisition: Wide angle X-ray scattering of polyamiline films were collected on a Scintag PAD X diffractometer with Bragg-Brentano geometry and an energy dispersive detector. Copper $K_{\alpha}$ radiation $(\lambda=0.1542 \mathrm{~nm}$ ) was used. The divergence, air scatter, acceptance and detector slits were $0.2,0.3,0.1,0.05$ $\mathrm{mm}$, respectively. Data were collected from 1.5 to 45 degrees $2 \theta$ in steps of 0.05 degrees for periods of $40-65$ seconds / step. The samples were made by stacking the films and approximate corrections were made for sample size, absorption and polarization.

SAXS scattering measurements were conducted at the $10 \mathrm{~m}$ facility ${ }^{5}$ at Oak Ridge National Laboratory (ORNL). The incident radiation in SAXS was copper $K_{\alpha}$ from a rotating anode source equipped with a monochromator. A $Q$ range of $0.05-4.5 \mathrm{~nm}^{-1}$ was used, where $Q=(4 \pi / \lambda) \sin \theta$ with $\lambda=0.154 \mathrm{~nm}$, the $X$-ray wavelength, and $\theta$, one half the scattering angle. Free standing isotropic film samples of $-6 \mathrm{~mm}$ diameter were cut and stacked to achieve a sample thickness of $-0.55 \mathrm{~mm}$. Samples were placedin a round multiple sample holder for transmission X-ray scattering. The data were then converted to an absolute scale by comparison with secondary standards ${ }^{6}$ and reported as differential cross sections per unit volume of sample, ( $1 \mathrm{\Sigma} / \mathrm{d} \Omega)$, with units of $\mathrm{cm}^{-1}$. Standard corrections for detector efficiency, beckground, empty cell scattering and sample transmission were applied. For comparison with wide angle X-ray scattering (WAXS) (at the same $\lambda$ ) this corresponds to a $2 \theta$ range of $0.07-6.3^{\circ}$. 
Results and Discussion

$X$-ray scattering of PANI-ES. Figure 1 shows WAXS diffractographs for several optimally doped polyanilines emeraldine salts (i.e. PANI-0.50-ES) and an undoped polyaniline emeraldine base (i.e. PANI-EB) cast from HFIP. In general, doping acts to change the crystalline order of the PANI-ES. This increase in order is apparently due to the increase in chain mobility. However, as seen in Figure 1, the relative increase in the degree of crystallinity from PANI-EB to PANI-ES is dependent upon the choice of dopant. Optimally doped PANI-0.50-HCSA salt shows the highest number of diffraction peaks compared to the other dopants surveyed.

Also seen in Figure 1 is evidence of the effect of interchain packing of polyaniline chains as a function of dopant counter-anion size. The smaller dopant, HMSA, shows a small diffraction peak at $8.9^{\circ}(2 \theta), d=0.99 \mathrm{~nm}$. This highlighted peak in Figure 1 shifts to smaller scattering angles as the counter-anion increases in size from HBSA $\left(5.0^{\circ}(2 \theta), d=1.8 \mathrm{~nm}\right)$ to HDBSA $\left(2.6^{\circ}(2 \theta), d=3.4 \mathrm{~nm}\right)$. It is thought that the respective PANIES contain regions of layers which are determined by the dopant counteranion size. The lack of well defined Bragg reflections in these PANI-ES samples does not permit a more detailed interpretation of chain ordering.

Since transport of charge carriers (i.e. chain hopping) occurs along the chain direction and between chains, it is likely that dopant induced changes in the polyaniline chain spacing are related to the observed (Figure 1) electrical conduction properties.

Scattering by PANI-ES / polycaprolactam blends. Figure 2 shows SAXS from PANI-ES / polycaprolactam blends. At the lowest values of $Q$ (i.e. $<0.5 \mathrm{~nm}^{-1}$ ) intense scattering indicates the presence of some additional component (i.e. perhaps small voids) in $100 \%$ polycaprolactam, PANI-ES and respective blends. In addition, the $100 \%$ polycaprolactam sample has a small shoulder at $Q \approx 0.9 \mathrm{~nm}^{-1}$ or $1.1^{\circ}(2 \theta)$ that corresponds to an $8 \mathrm{~nm}$ periodicity. This implies that the host material is semi-crystalline when cast from HFIP solvent. The $\sim 40 \%$ (vol/vol) blends are seen to suppress the $8 \mathrm{~nm}$ periodicity between the lamellae crystals. However, the degree to which this long-range order is suppressed is seen to be dependent upon the functionality of the dopant counter-anion. For example, the $41 \%$ loading of PANI-0.50-HDBSA shows some remnants of the peak corresponding to the $8 \mathrm{~nm}$ lamellae spacing; however, the $41 \%$ PANI-0.50-HCSA completely suppresses the peak. This suggests that the HCSA dopant anion is interacting with the polycaprolactam to a greater extent. This is primarily due to a combination of an increase in hydrogen bonding interactions and similarities in polarity between the two blend components. The same trend is found in the $20 \%$ ( $\mathrm{vol} / \mathrm{vol}$ ) loading of the respective salts in polycaprolactam.

The WAXS diffractograph in Figure 3 e shows that the polycaprolactam crystal structure is primarily the $\alpha$ form ${ }^{7}$ when solution cast from HFIP. As seen in Figures 3-a to 3-d the host polymer displays two large diffraction peaks that do not shift as a function of dopant counter-anion structure. This suggests that the short-range order (i.e. the unit cell) in the polycaprolactam matrix is not perturbed by the respective PANI-ES.

Even though the SAXS shows an increasing amount of disorder between lamellae crystals as a function of PANI-ES content, the WAXS displays a large amount of crystallinity left in the polycaprolactam. This suggests that there are regions of incomplete mixing between the two components and the conductive blends surveyed are characterized as a phase-separated system. 
Light microscopy. Blends of PANI-0.50-HMSA and PANI-0.50HDBSA with polycaprolactam showed some level of phase segregation at volume fractions of less than $1 \%$ of PANI-ES. As seen in Figure 4, the micrographs of the PANI-0.50-HDBSA domains are globular, whereas the more polar dopant, PANI-0.50-HMSA yields a more expanded, continuous morphology in the same polycaprolactam matrix. The nature of the dual phase behavior in both of these blends is thought to be due to the differences in conformation between the semi-rigid PANI-ES and the flexible polycaprolactam and differences in dopant polarity. We propose that the resulting blend morphology in the micrographs stems from the different degrees of compatibility between each respective salt in the polycaprolactam. From Figure 4, the lower conductivities in the PANIHDBSA blend parallel a coarser, more aggregated morphology whereas the higher conductivities accompany a finer, more dispersed network in the PANI-HMSA blend.

\section{Acknowledgment}

This research was in part supported by a Research Opportunity Award of Research Corporation and by the Division of Materials Sciences, Office of Basic Energy Sciences, Department of Energy, under contract DE-AC05960R22464 with Oak Ridge National Laboratory, managed by Lockheed Martin Energy Research Corp.

(1) (a) Cao, Y.; Smith, P.: Heeger, A.J. Synth. Metals, 1992, 48, 91.

(2) Zallen, R. The Physics of Amorphous Solids, John Wiley: New York,1983; Chapter 4.

(3) Reghu, M.; Yoon. C. O.; Yang, C. Y. Moses, D.; Smith. Paul; Heeger, A. J.; Cao, Y. Phys. Rev. B. S 0, 19, 13931-13941.

(4) Hopkins, A. R.; Rasmussen, P. G.; Basheer. R. A.; Mocromolecules. 1996, 29. 7838-7846.

(S) Wignall, G. D.; Lin. J.S.; Spooner, S. J. Appl. Crystal. 1990, 23, 241.

(6) Russel, T.P.; Lin, J.S.; Spooner, S.; Wignall, G.D. J. Appl. Crystal.. 1988, 21, 629.

(7) Parker, James P.; Lindenmeyer, Paul H. J. Appl. Polym. Sci. 1977, 21, 821 837. 


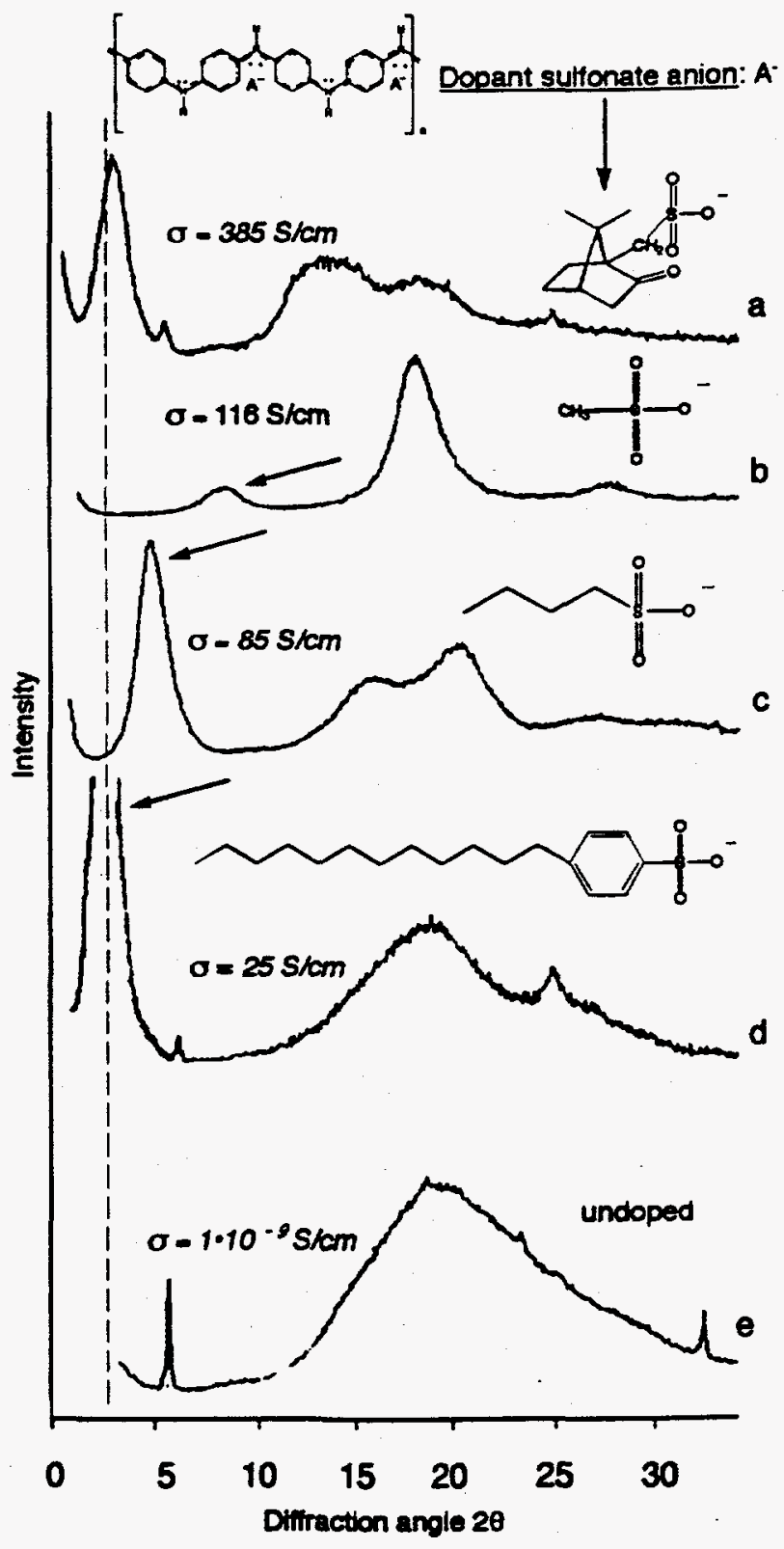

Fure 1. Wide mole X-my memerine (WAXS) of froe anding, optim illy dopod film can from herefluoro-2-propmed: (o) PANI-0.5O-HCSA; (b) PANI-0.SO-KMSA; (c) PANI-0.SO-KBSA; (d) PANI0-SO KDBSA and andoped (c) PANI-EB. 


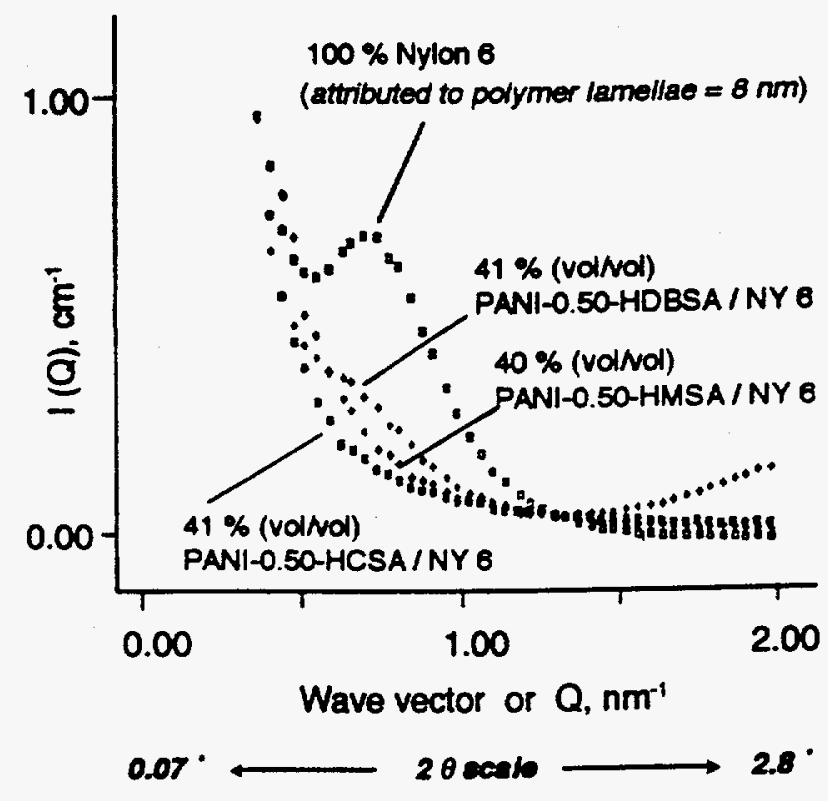

Fape 2 Small angle X-rny ecattering (SAXS) of poibmiline i mylon 6 blende. Fitem ere cat from bexuluoro-2-propanol. 


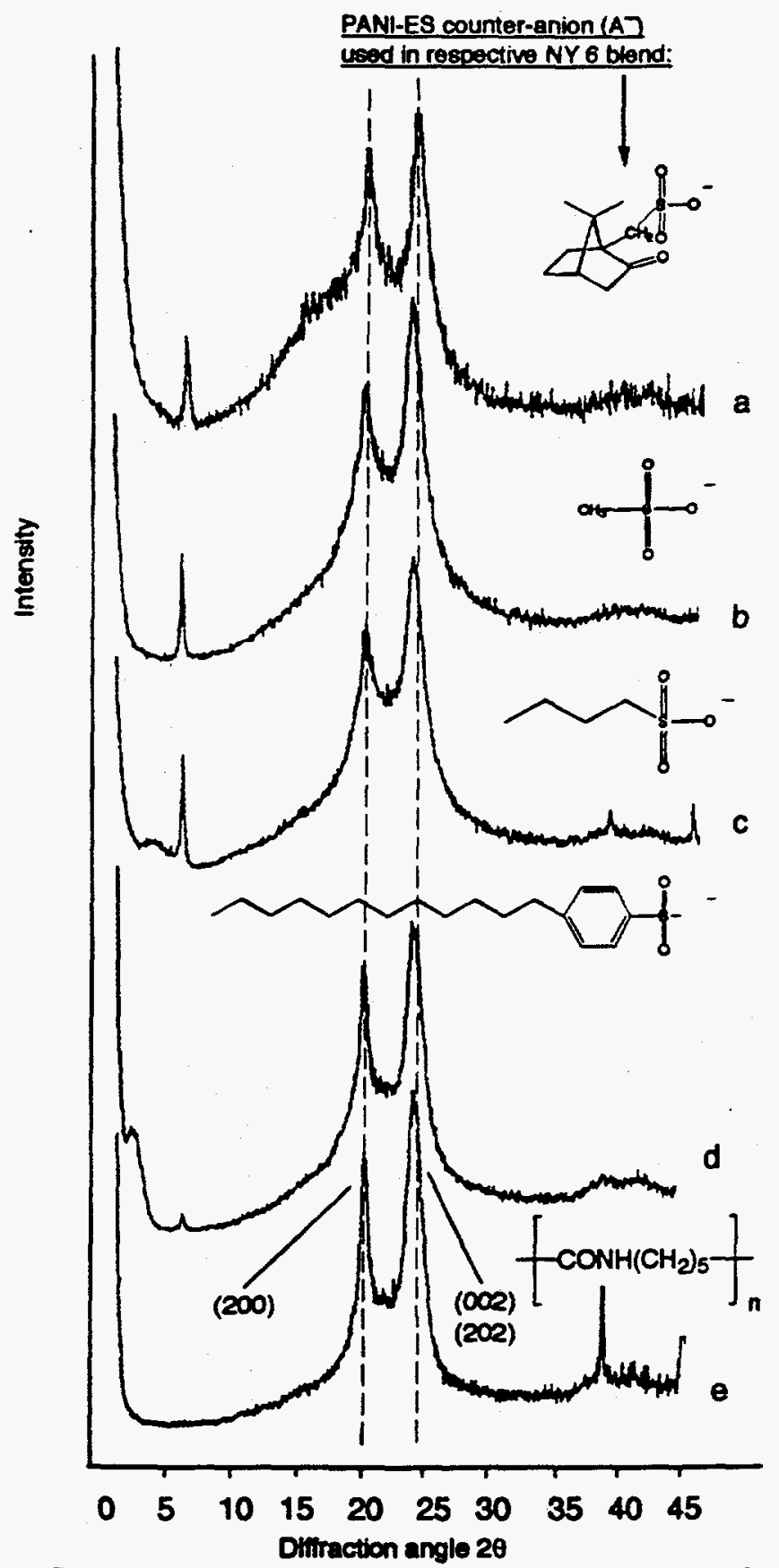

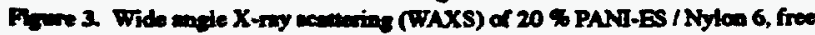

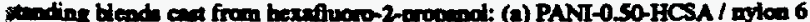
(b) PANI-0.SO-HMSA / aylon 6; (c) PANT-0.SO-HIBSA / ayion 6; (d) PANI-0.50 BDBSN ayion 6; (e) $100 \%$ myion 6. 


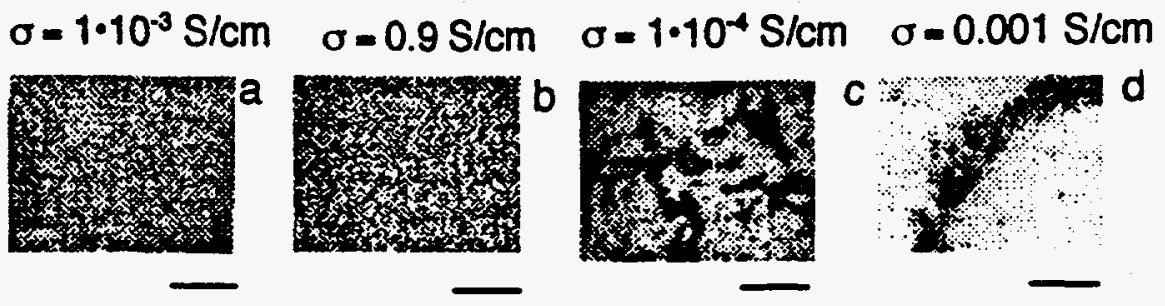

Flzare 4. Light microscopy micrographs of blends cast from hexafluono-2-propanol: (a) $0.5 \%$ (vol/vol) PANI-0.50-HMSA / nyion 6; (b) $10 \%$ (vol/vol) PANI-0.50-HMSA I nylon 6; (c) $0.5 \%$ (vol/vol) PANI-0.50-HDBSA / nylon 6; (d) $10 \%$ (vol/vol) PANI$0.50-H D B S A /$ nylon 6. Dark areas are PANI-ES. 